passes zero in one degree of freedom at the tunnel exit ${ }^{8}$. This acknowledges the notion that pure tunnelling dynamics in a semiclassical description is characterized by an imaginary momentum component in at least one degree of freedom and by the fact that all momenta are real in classically allowed regions.

Using classical backpropagation of an ionized wave packet (determined quantum mechanically by solving the Schrödinger equation), one arrives at the conclusion that the tunnelling time defined with this tunnel exit is zero for single-electron dynamics ${ }^{9}$. The same conclusion has been reached with different techniques ${ }^{10}$ and certainly corroborates our 'quantum' intuition that for a single electron under a potential barrier, no (real) time passes.
At least two caveats remain for this intuition: it ignores interference and diffraction phenomena that may complicate determining tunnel exits in a meaningful way, and secondly, it is only valid for a single (or independent) electron ruled by a potential. Substantial interaction with other electrons will imprint an additional phase on the tunnelling electron that may provide a timescale for tunnelling referenced by the remaining electron(s). This renders our earlier statement that experiments cannot measure tunnelling times because they do not exist as premature. Nevertheless, thanks to the beautiful attoclock experiments in helium and now in hydrogen, we can be sure that a reasonably defined tunnelling time is zero in these cases. Yet, the legacy of tunnelling will continue.
Jan M. Rost (D)* and Ulf Saalmann

Max Planck Institute for the Physics of Complex Systems, Dresden, Germany.

*e-mail: rost@pks.mpg.de

Published online: 21 June 2019

https://doi.org/10.1038/s41566-019-0472-9

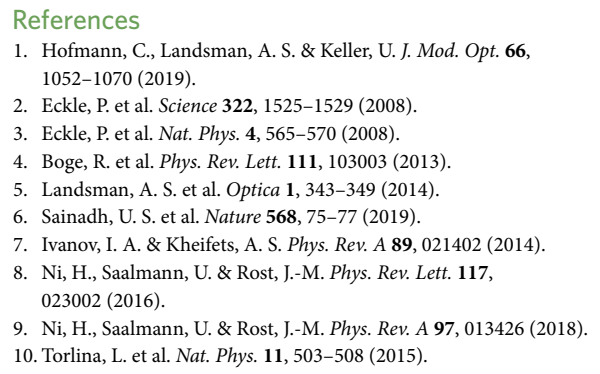

\title{
DISPLAYS
}

\section{Tunable plasmonic pixels}

Colour displays based on nanoplasmonics offer some advantages over conventional dye- or structural-based approaches. However, rapid and energy-efficient tuning of colours in plasmonic displays remains a challenge. Now, Jialong Peng, Hyeon-Ho Jeong and colleagues at Cambridge University, UK, have demonstrated a nanoplasmonic display with pixels that show tuning of resonance wavelength (colour), rapidly $(>50 \mathrm{~Hz})$ and over a huge $100 \mathrm{~nm}$ range (at visible wavelengths) (J. Peng et al., Sci. Adv. 5, eaaw2205; 2019). Importantly, the tuning is energy efficient, with each pixel taking only $0.2 \mathrm{fJ}$ per $1 \mathrm{~nm}$ wavelength shift.

Au nanoparticles (NPs) sit on an Au substrate (which is also the working electrode), but with carefully controlled particle-mirror spacing. The particles are electrochromic thanks to encapsulation in a conductive polymer shell (polyaniline); the shell also precisely dictates the particle-mirror gap and is tailored via bottom-up processing. The particles are randomly dispersed onto the planar $\mathrm{Au}$ substrate, but coverage can be engineered and the structures are incorporated into custom-built electromechanical cells, enabling tracking of both electrical dynamics and optical response (via darkfield microscopy; see image). The chemical state (and optical properties) of the shells is modified by applied voltage, swept from -0.2 to $0.6 \mathrm{~V}$ (at $50 \mathrm{mV} \mathrm{s}^{-1}$ ). The resulting $\sim 100 \mathrm{~nm}$ resonance shifts are not only reversible but the colour and

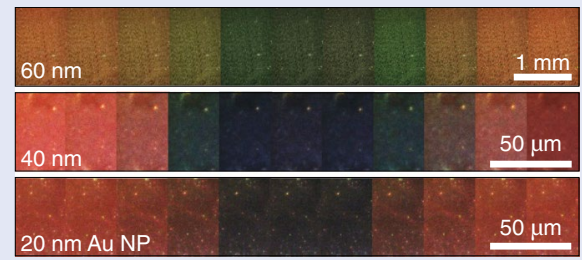

Credit: Modified from Peng et al. (c) The Authors, some rights reserved; exclusive licensee AAAS. Distributed under a Creative Commons License (http://creativecommons.org/licenses/by$\mathrm{nc} / 4.0 /$ ). Reprinted with permission of AAAS

structures are shown to be stable for at least three months.

The corresponding author, Jeremy Baumberg, told Nature Photonics that they had previously been trying to make structural colour materials, using nanoassembly on a large scale. They had some success but realized that making electrically tunable wallpapers was going to require a lot of energy if using bulk structures, so they turned to surface photonics. Earlier work in collaboration with Nokia used holography to make deformable metallic structures like kirigami.

"We can make a scalable coating that allows switching of colour of a film with extremely low energy, which opens up the possibility of building-scale displays," Baumberg explained. "Scientists have been making plasmonic pixels made of noble metals for the last decade, but these all have fixed colours (the idea being to make permanent coloured images that don't fade). But switching in this way is new. Our display also can be viewed from any angle, and in any light conditions (except darkness) since it is a scattering-based colour which doesn't require a backlight."

The work was not without hurdles. Baumberg told Nature Photonics that it's a challenge to optimize all of the parameters at the same time. For example, it's not simple to make good blue colours, while keeping costs low, everything flexible, and with good electrical properties, and so on.

"It shows a nice route to making nanophotonics devices that can be scaled onto roll-to-roll processing, which is what I have been trying with a team for a few years," Baumberg stated. "I've been tired of seeing high-cost nanofabrication used for devices with the comment that it could be transferred as generally such high-accuracy nanostructuring is a big roadblock. Using solution growth is really important."

Key to the result, according to Baumberg, was the ability to trap light into tiny gaps, meaning that low energy is required for tuning due to the small volume of material where the optical properties need to be changed. The team is now looking to scale up to larger multipixel demonstrators, as well as to push the colour range.

\section{David F. P. Pile}

Published online: 21 June 2019

https://doi.org/10.1038/s41566-019-0478-3 